CASE REPORT

\title{
Gait and posture assessments of a patient treated with deep brain stimulation in dystonia using three - dimen- sional motion analysis systems
}

\author{
Shigetaka Nakao', Koji Komatsu², Waka Sakai ${ }^{3}$ Michiharu Kashihara', \\ Yuki Masuda², Koji Nishikawa², Tetsuya Okahisa², Shin Kondo², Toshifumi Osawa², \\ Ryuji Kaji ${ }^{4}$, Satoshi Gotoh ${ }^{4}$, Shinji Nagahiro ${ }^{5}$, Shinjiro Takata ${ }^{1}$, and Natsuo Yasui ${ }^{1}$ \\ ${ }^{1}$ Department of Orthopedics, Institute of Health Biosciences, the University of Tokushima Graduate \\ School, ${ }^{2}$ Division of Rehabilitation, ${ }^{3}$ Department of Neurology, Tokushima University Hospital, ${ }^{4}$ Depart- \\ ment of Clinical Neuroscience, and ${ }^{5}$ Department of Neurosurgery, Institute of Health Biosciences, \\ the University of Tokushima Graduate School, Tokushima, Japan
}

\begin{abstract}
Kinesiologic analysis of gait disorders, postural instabilities and abnormal movements is quite difficult to assess objectively by clinical observation, such as by specific scale and video recordings. In this study, we reported one of the aspects of the usefulness of three-dimensional motion analysis (Vicon Systems, Oxford, United Kingdom), which can measure inclusive data of movement disorders and substitute for conventional assessments. A 49-year-old man who had various dystonic symptoms, mainly on his left side of the body, responded well to deep brain stimulation (DBS). The examination quantified how the involuntary movements or other symptoms with dystonia changed before and after treatments. J. Med. Invest. 58 : 264-272, August, 2011
\end{abstract}

Keywords : motion analysis, dystonia, DBS

\section{INTRODUCTION}

Involuntary movements of dystonia interfere with normal posture and gait. Abnormal posture and gait disturbance are associated with frequent falls and restrict activities of daily living (ADL), which can reduce the quality of life (QOL). Deep brain stimulation (DBS) is a safe and successful therapeutic option for patients with gait disturbance in Parkinson's disease and dystonia. Globus Pallidus internus (GPi) DBS is effective for the treatment of generalized

Received for publication May 12, 2011 ; accepted June 28, 2011.

Address correspondence and reprint requests to Shigetaka Nakao, Department of Orthopedics, Institute of Health Biosciences, the University of Tokushima Graduate School, Kuramoto-cho, Tokushima 770-8503, Japan and Fax : +81-88-633-9313. dystonia (1). GPi DBS for X-linked dystonia improves $67.9-80.6 \%$ of symptoms (2-5).

Functional neurological impairment has been measured by specific observational analysis, such as a rating scale, video monitoring, conventional three-dimensional kinematic recordings using multiple videos, electromyography and electro-goniometry for the analysis of gait, a force plate for the analysis of postural stability, and multiaxial accelerometers and gyroscopes for the analysis of movement disorders. Conventional three-dimensional kinematic recordings are complicated and time consuming, and it is difficult to extract data (6). The Vicon system is a simplified three-dimensional motion analysis system that integrates conventional approaches. Vicon motion measurements have been used in clinical and research laboratories combined with 
an MX camera, which captures three-dimensional optical marker-based technology, to provide inclusive motion data in detail. We used the Vicon system to assess a patient with generalized dystonia before and after the implantation of GPi DBS electrodes. In this paper, we present the outcome of a case of dystonia treated with DBS to show its therapeutic efficacy to improve postural alignment and gait disturbance.

\section{CASE REPORT}

\section{Patient presentation}

A 49-year-old Filipino male, native to the island of Panay, was admitted to our hospital to treat dystonia. He had been previously diagnosed with Xlinked dystonia-parkinsonism (DYT3 ; "lubag" disease) $(7,8)$. He first presented with involuntary movements of his bilateral halluxes at the age of 41 . He later developed involuntary movements of his upper and lower limbs, cervical dystonia and spinal torsion causing gait disturbance and abnormal posture at the age of 43 .

His ambulation progressively worsened. The baseline United Dystonia Rating Scale (UDRS) score performed at our institution was $64 / 5 / 112$, and his Burk-Fahn-Mardsden Scale was 57/120 (9). He was found to have dystonic symptoms mainly on the left side of his body with a retracted head, twisted trunk and involuntary movements of his upper limbs. He also had difficulties with vocalization, swallowing and eye opening. His dystonia was very disabling, greatly affecting his quality of life (QOL).

\section{Experimental design}

Motion analysis using the Vicon system was conducted to record kinematic and kinetic data during static posture and dynamic walking. The patient was evaluated in three-dimensional static posture and consecutive gait using the Vicon MX system (Vicon Motion Systems, Oxford, United Kingdom). Kinematic data were collected at $120 \mathrm{~Hz}$ using a passive eight-camera system (Vicon MX T20 ; Vicon Motion Systems). Kinetic parameters were recorded at 120 $\mathrm{Hz}$ using a four-embedded ground force platform (AMTI, model OR-06 ; Advanced Mechanical Technology, Watertown, MA). Kinematic and kinetic systems were synchronized for simultaneous collection. Nexus 1.4 software (Vicon Motion Systems) derived the kinematic and kinetic parameters of standing for thirty seconds and five times walking on a ten-meter walkway.

Markers (14-mm diameter) which reflected infrared rays were placed on landmarks of the whole body following the Plug-in-Gait model (Fig. 1, Vicon Motion Systems). Eight MX cameras captured the motion of markers and Nexus 1.4 processed the motion data as stick images from marker positions in three dimensions. Nexus 1.4 extracted c3d data (three-dimensional coordinated data of model) extracted from the plug-in-gait model parameters. Polygon 3.1 software (Vicon Motion Systems) simulated the human motion (skeletal model) expressed by the rigid body of the plug-in-gait model from c3d data. In addition, Medicaptures (Winpod, Balma, France) were used to record the distribution of foot pressure and the tracks of length (LNG) by postural sway of the center of mass (COM) in static posture, which showed the stability of static balance. He was evaluated before and after DBS (16 days after DBS) using Vicon. He was examined using various parameters such as neck angle (the angles between the head relative to the thorax) for cervical dystonia and spine angle (the angles between the thorax relative to the pelvis) for spinal torsion, COM changes for the stability of dynamic balance, patterns of ground reaction force (the force exchanged between the foot and the ground while walking) for symmetrical motion of limbs, and gait parameters (cadence, speed, step

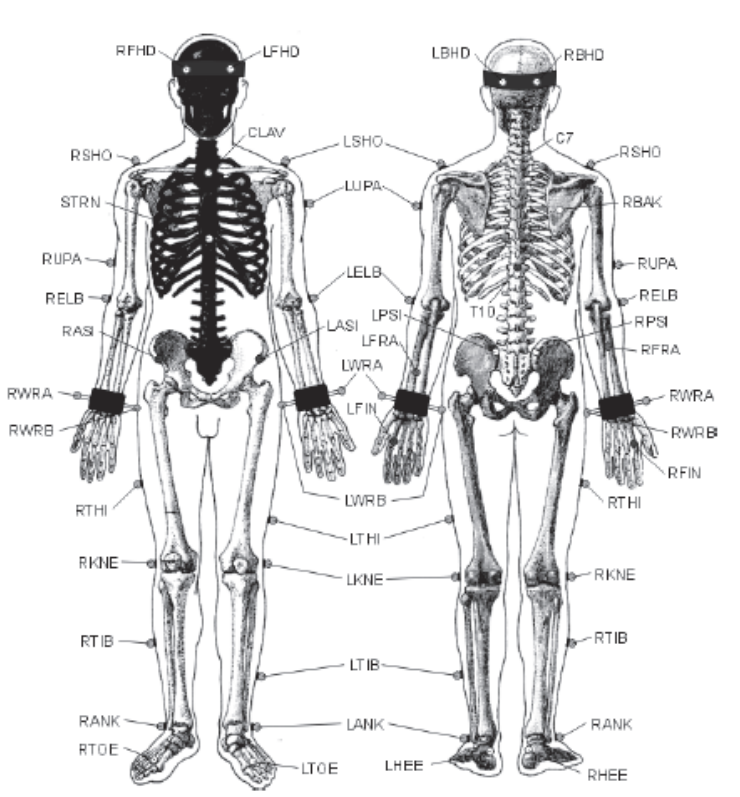

Figure 1. Plug-in gait marker placement

Thirty-five reflective markers were placed on landmark of the whole body. The distribution of markers consisted of head (four), trunk (nine), upper limbs (fourteen) and lower limbs (ten). 
length, step width, single support time) for gait disturbance or symmetrical gait. He maintained a static posture for 30 seconds and gait five times and the mean values were calculated. All gait data were normalized by the gait cycle.

\section{RESULTS}

\section{Posture}

\section{1-1) Foot pressure and COM}

Skelton models confirmed the external differences in static posture (Fig. 2). The patient's model before DBS inclined to the left side with poor position of his neck and trunk. His right foot pressure was distributed to his toe and heel (forefoot 51\% and hindfoot $49 \%$, average $415 \mathrm{~g} / \mathrm{cm}^{2}$ ) and the left was deviated to his heel (forefoot $19 \%$ and hindfoot
$81 \%$, average $543 \mathrm{~g} / \mathrm{cm}^{2}$ ). The LNG, which showed postural instability, was $923.2 \mathrm{~mm}$. After DBS, distribution of foot pressure was on the right side (forefoot $65 \%$ and hindfoot $35 \%$, average $361 \mathrm{~g} / \mathrm{cm}^{2}$ ) and on the left (forefoot $18 \%$ and hindfoot $82 \%$, average $510 \mathrm{~g} / \mathrm{cm}^{2}$ ), and the LNG decreased to $502.9 \mathrm{~mm}$. The balance of weight bearing and right-left ratio of foot pressure showed no difference between before and after DBS. LNG revealed clear shortening of $54.5 \%$ and shifted to the middle of his feet after DBS.

1-2) Neck and spine angle

His spine had extended to the left side, bent and rotated to the left before, and was slightly bent and rotated to the left after DBS (Fig. 3). His neck, however, had extended to the right side, bent and rotated to the right before, and was slightly bent and rotated to the left after DBS. His postural alignment
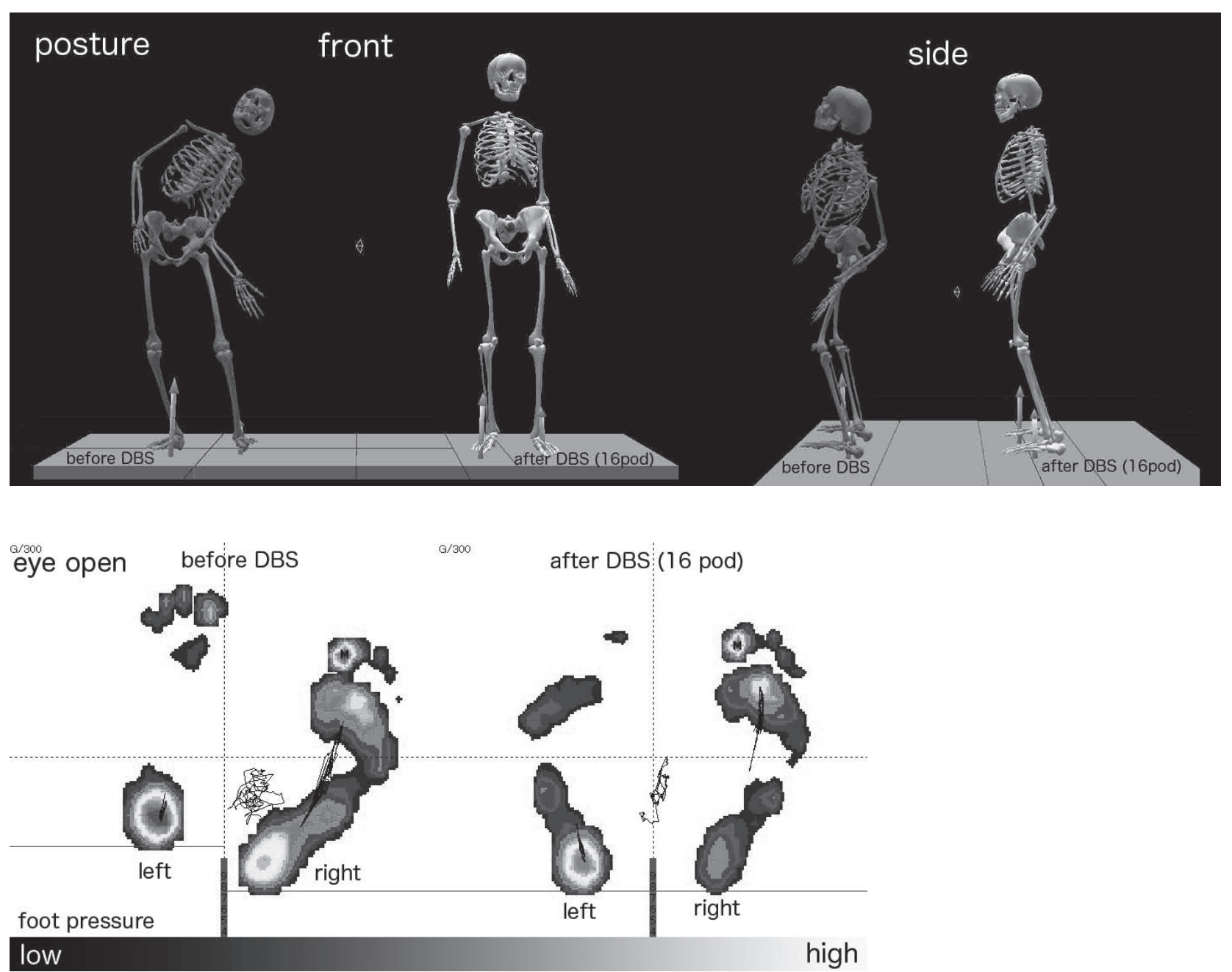

Figure 2. Static posture and foot pressure

Skelton models from Polygon 3.1 simulated and visualized the postural alignments in frontal and sagittal planes. The patient stood on force plates with the eyes open and relaxed. Abnormality before DBS (left) especially showed spinal torsion with left postural deviation. The lines of the center of pressure showing postural instability improved on middle of the foot images after DBS. 
Neck angle
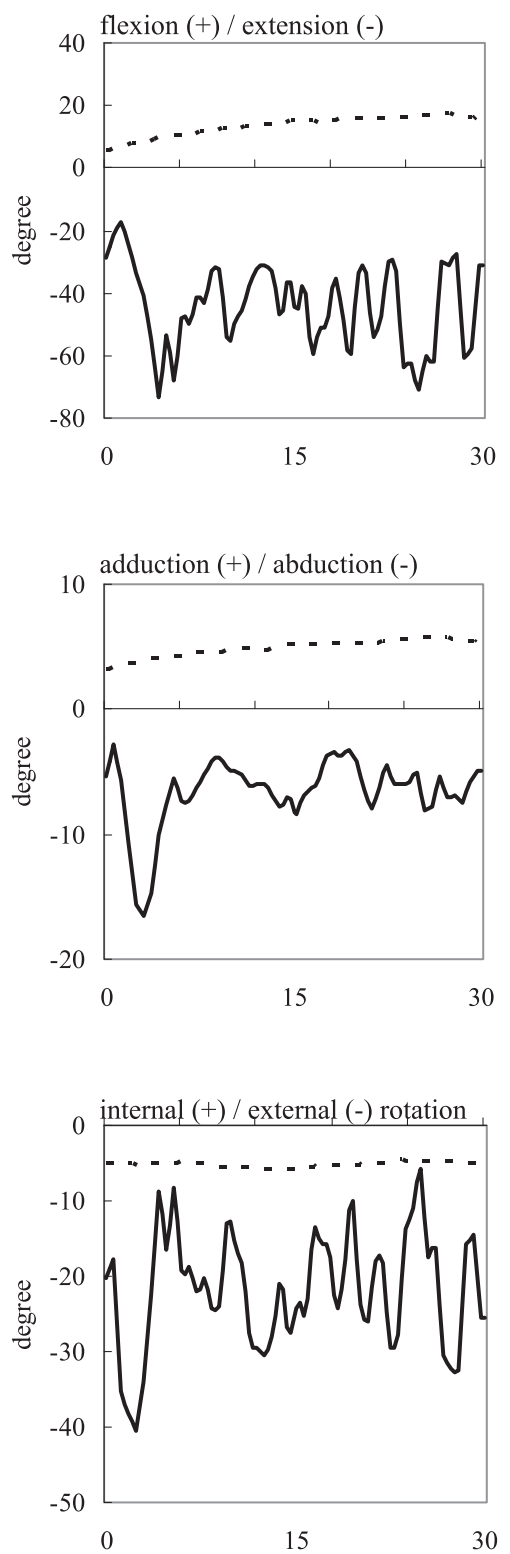

Spine angle
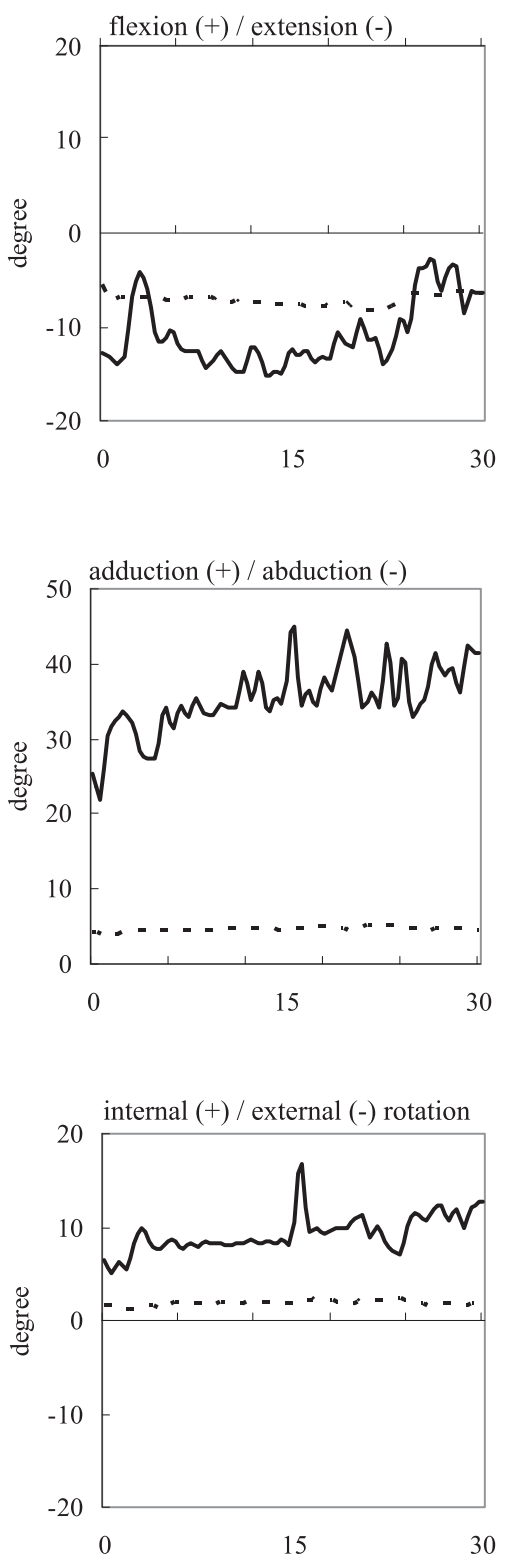

after DBS

Figure 3. Neck and spine angle in static posture

The degree of neck and spine angles with static standing for thirty seconds improved to maintain a stable position after DBS in three dimensions. Neck movements before DBS were antagonists of those of the spine in lateral bend and vertical directions ; these symptoms decreased after DBS.

remained almost straight (offset) from the center of his body (0 degrees) after DBS (neck offset : flexion $13.5 \pm 3.4$ degrees, left bending $4.9 \pm 0.8$ degrees, right rotation $5.1 \pm 0.7$ degrees, spine offset : flexion $13.3 \pm 2.8$ degrees, left bending $4.7 \pm 0.3$ degrees, left rotation $1.8 \pm 0.8$ degrees).

\section{Gait}

\section{2-1) Gait parameters}

The changes in gait parameters are shown in
Table 1 . The following parameters were also compared with normal data (normal values) (10-12). All values after DBS were better than before but could not reach normal values for increasing cadence (110-120 steps/min), faster walking speed $(1.36 \mathrm{~m} /$ sec), longer step length $(0.65 \mathrm{~m})$, shorter step width and single support time.

2-2) Neck and spine angle

The alignments of his spine before, which extended, bent and rotated to the left, changed to a 
Table 1. Gait parameters

\begin{tabular}{lcccc}
\hline & \multicolumn{2}{c}{ Before DBS } & \multicolumn{2}{c}{ After DBS } \\
\hline Cadence (steps/min) & Left & Right & Left & Right \\
Walking speed (m/sec) & $93.8 \pm 13.0$ & $91.3 \pm 18.3$ & $100.0 \pm 8.79$ & $92.7 \pm 5.83$ \\
Step lengths (m) & $0.65 \pm 0.09$ & $0.62 \pm 0.20$ & $0.86 \pm 0.15$ & $0.86 \pm 0.05$ \\
Step width (m) & $0.26 \pm 0.10$ & $0.54 \pm 0.14$ & $0.45 \pm 0.18$ & $0.58 \pm 0.05$ \\
Single support (sec) & $0.30 \pm 0.04$ & $0.28 \pm 0.02$ & $0.20 \pm 0.05$ & $0.21 \pm 0.03$ \\
\hline
\end{tabular}

Values are expressed gait parameters (mean \pm SD) for five times walking. The parameters became more symmetry after DBS.

lower degree of spinal deviation close to a straight position on gait after DBS (Fig. 4). His neck angles after DBS were still abnormal (flexion, left bending and right rotation) even after DBS. The standard deviations of each angle stayed in the lower ranges, showing stability during a gait cycle.
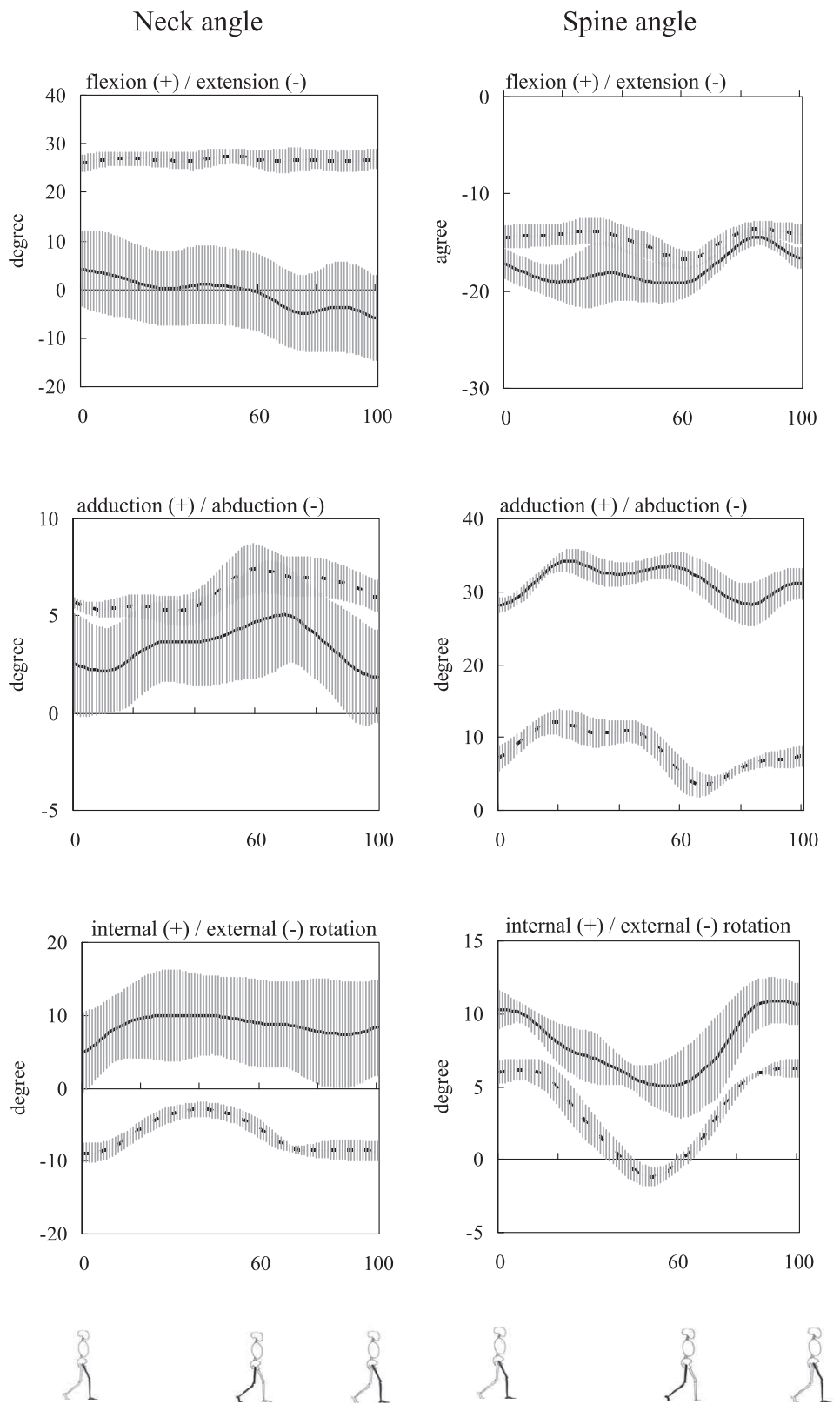

before DBS after DBS

Figure 4. Neck and spine angles during a gait cycle

Angles (mean \pm SD, degree) of the patient with five times gait. After DBS, the spine angle became close to the median line of the body with moderate standard deviation in all planes (sagittal, frontal and coronal planes). Neck position remained inclined to the left. 
2-3) Ground reaction force (GRF)

The asymmetry of GRF patterns (Fig. 5) before DBS became close to symmetrical after DBS (lateral shear and vertical forces), except for a progressive pattern. Each lower limb had a respective role in braking force for the left and driving force for the

Ground reaction force
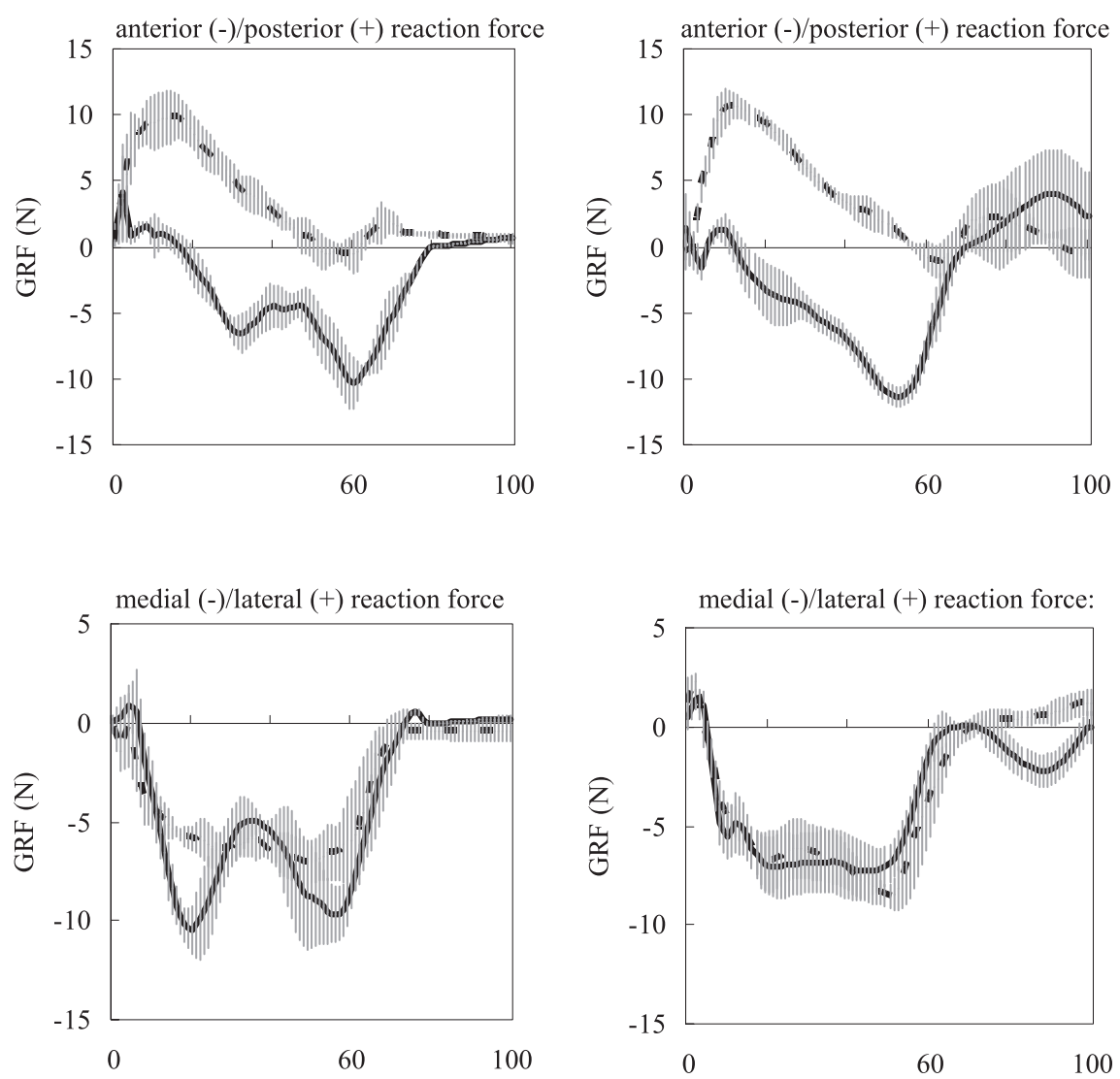

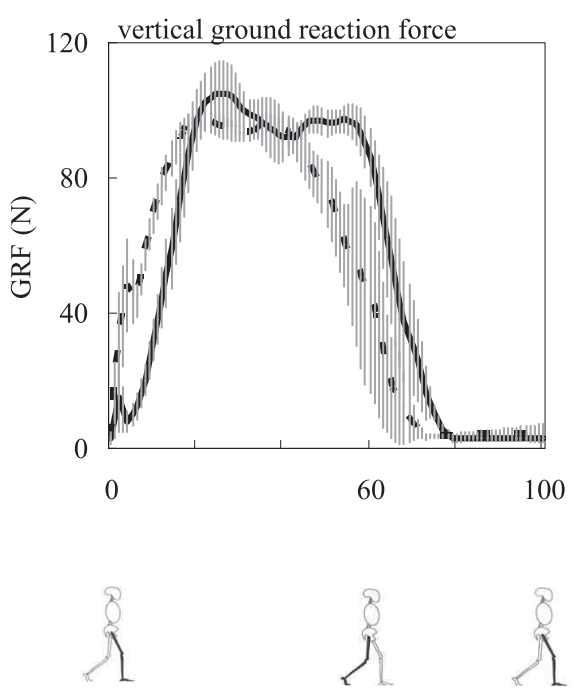

before DBS

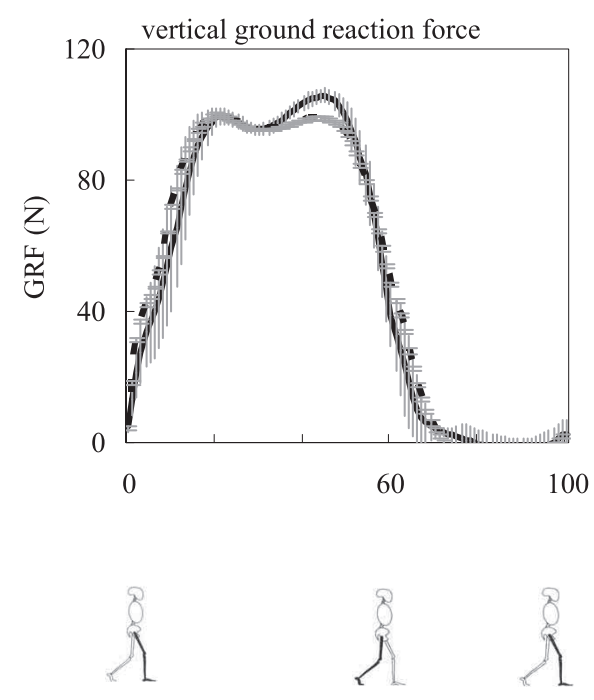

after DBS

left side right side

Figure 5. Ground reaction force during a gait cycle

GRF (mean \pm SD, Newton) from force plates with five times gait showed close to symmetrical bilateral gait patterns after DBS, except progressive shear forces. Dystonia side (left) reversed normal side (right). 
right in progressive shear force.

2-4) Center of mass (COM)

The ranges (distance) of COM were, before DBS : $173.4 \pm 26.7 \mathrm{~mm}$ (lateral : L), $66.5 \pm 22.4 \mathrm{~mm}$ (vertical : V) and after : $70.5 \pm 17.2 \mathrm{~mm}(\mathrm{~L}), 22.5 \pm$ $4.8 \mathrm{~mm}(\mathrm{~V})$. The normal values were $58.0 \pm 20.0$ $\mathrm{mm}(\mathrm{L})$ and $48.0 \pm 11.0 \mathrm{~mm}(\mathrm{~V})(10)$. The deviation of COM revealed stability in both lateral and vertical directions during a gait cycle (Fig. 6).

\section{DISCUSSION}

The mechanism of the DBS effect was associated with the disruption of pathological network activity in the cortico-basal ganglia-thalamic circuits by affecting the firing rates and bursting patterns of neurons and synchronized oscillatory activity of neuronal networks (13). There is a consensus that idiopathic generalized, cervical and segmental dystonia

\section{Center of mass displacement}
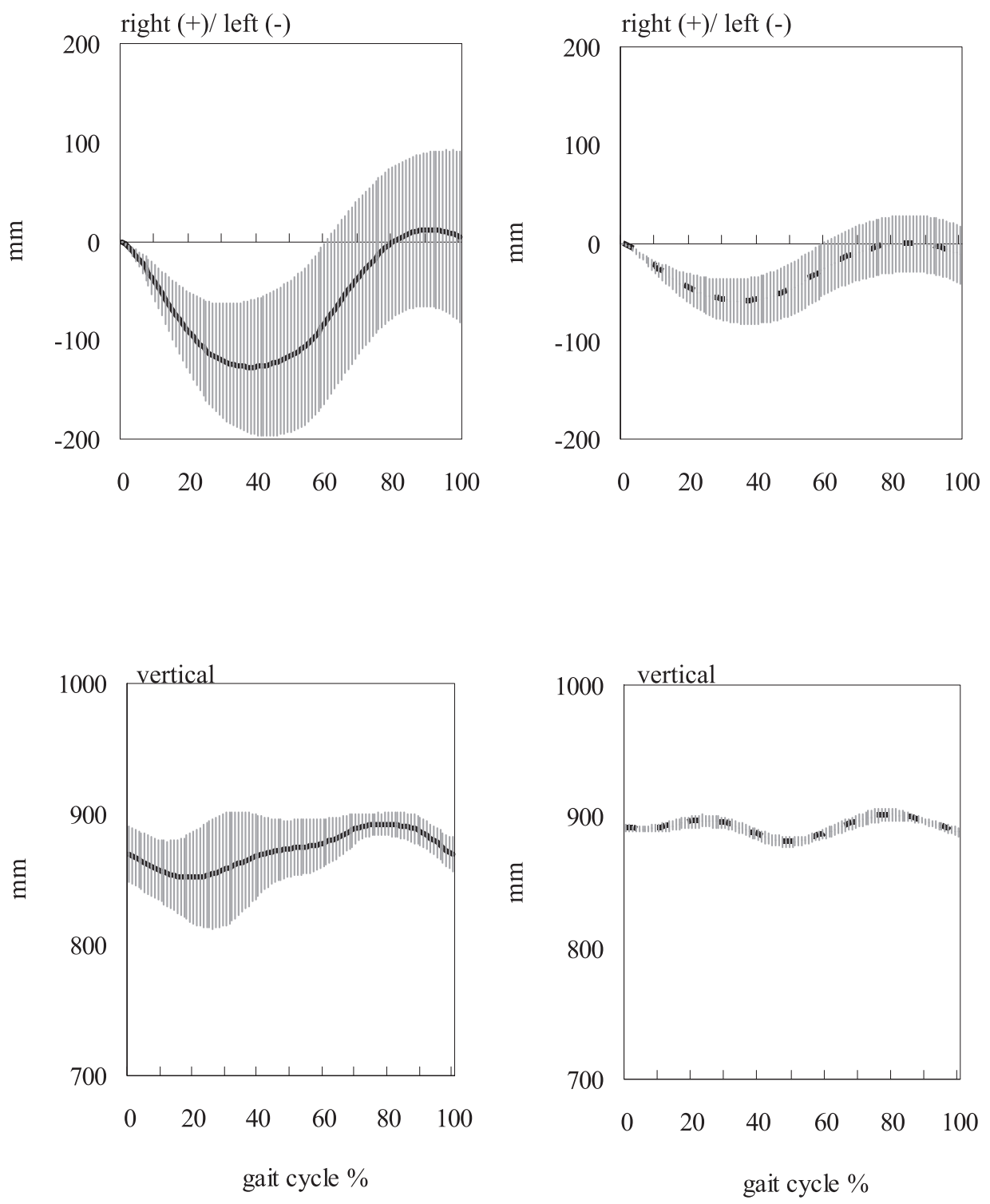

before DBS

after DBS

Figure 6. COM patterns during a gait cycle

Displacement of center of mass (mean \pm SD, mm) with five times gait in lateral and vertical directions for gait instability; wide ranges of standard deviations before DBS revealed less stability than after DBS during the gait cycle. 
are good indications of DBS and efficacy is maintained long term. Although pallidal DBS has been shown to be cognitively safe, non-dystonic extremities have not received much attention (14). We clarified which involuntary movements were related to postural instability and gait disturbance by DBS in a dystonic patient using three-dimensional motion analysis in this study. His posture and gait were asymmetrical and unstable before DBS ; therefore, he quickly became exhausted easily and fell down frequently (15), but they improved close to symmetrical after DBS. Functional body balance was controlled by changes of symptoms (with partial corrections of neck and spinal alignments in a static posture) and maintained the stability of COM and COP. His neck angles remained abnormal with specific motions during gait compared to the spine, which was not disturbed in walking. Functional improvements of gait, such as gait parameters including increasing of cadence (step rate) and walking speed, increased step length, reduction of a wide base, extension of single support time and symmetrical GRF patterns in lateral and vertical shear force close to normal patterns in consecutive gait showed dynamic stability simultaneously. Gait needs the neuromuscular function of the whole body and involves involuntary as well as voluntary motor elements (11). DBS facilitated the possible relations of gait asymmetry to postural instability in dystonia as well as Parkinson's disease (16). These improvements of gait parameters also explained that of neuromuscular function, which well responded to DBS. Not all symptoms could be treated with DBS (2-4). Our patient improved $80 \%$ by BFMS with slightly abnormal movement in his posture and gait. Some dependence on the right side remained for weight bearing to substitute the symptomatically dominant side during gait even after DBS. Other symptoms, including slightly better vocalization, swallowing and keeping his eyes open could help the patient to be independent and active during hospitalization. These remaining symptoms will necessitate a rehabilitation program, using training to establish new movement patterns, to preserve an appropriate activity level, and to treat the specific disability, which resulted from secondary changes of the musculoskeletal system during pathological muscle tension in dystonia (17) after DBS.

The results of this study revealed that threedimensional motion analysis could inclusively assess the level of improvement in a patient with movement disorders and could assist in diagnosis or effect measurement. We also need to collect more data from patients with movement disorders and clarify their specific characteristics in future studies.

\section{REFERENCES}

1. Sako W: Gait disturbance and deep brain stimulation. Brain Nerve 62(11) : 1221-1225, 2010 (in Japanese)

2. Evidente VG, Lyons MK, Wheeler M, Hillman R, Helepolelei L, Beynen F, Nolte D, Müller U, Starr PA : First case of X-linked dystoniaparkinsonism ("Lubag") to demonstrate a response to bilateral pallidal stimulation. Mov Disord 22(12) : 1790-1793, 2007

3. Martinez-Torres I, Limousin P, Tisch S, Page R, Pinto A, Foltynie T, Bhatia KP, Hariz MI, Zrinzo L: Early and marked benefit with GPi DBS for Lubag syndrome presenting with rapidly progressive life-threatening dystonia. Mov Disord 24(11) : 1710-1712, 2009

4. Wadia PM, Lim SY, Lozano AM, Poon YY, Diaz CT, Moro E : Bilateral pallidal stimulation for X-linked dystonia parkinsonism. Arch Neurol 67(8) : 1012-1015, 2010

5. Oyama G, Fernandez HH, Foote KD, Zeilman P, Hwynn N, Jacobson CE $4^{\text {th }}$, Malaty IA, Rodriquez RL, Okun MS : Differential response of dystonia and parkinsonism following globus pallidus internus deep brain stimulation in Xlinked dystonia parkinsonism (Lubag). Stereotact Funct Neurosurg $88: 329$-333, 2010

6. Blanc Y, Merio A, Landis T, Burkhard PR: Kinesiologic analysis of gait and abnormal movements. Rev Med Suisse Romande 123 : 327-33, 2003

7. Müllar $\mathrm{U}$ : The monogenic primary dystonias. Brain 132(8) : 2005-2025, 2009

8. Evidente VG, Nolte D, Niemann S, Advincula J, Mayo MC, Natividad FF, Müller U : Phenotypic and molecular analyses of X-linked dystoniaparkinsonism ("lubag") in women. Arch Neurol 61(12) : 1956-1959, 2004

9. Comella CL, Leurgans S, Wuu J, Stebbins GT, Chmura T : Rating scales for dystonia : A Multicenter Assessment. Mov Disord 18:303-312, 2003

10. Oberg T, Karasznia A, Oberg K : Basic gait parameters : Reference data for normal subjects, 10-79 years of age. J Rehabil Res Develop 30 : 210-223, 1993 
11. Nakamura R, Saitoh H : Fundamental Kinesiology fifth edition, chapter 8, Ishiyaku Publishers, Inc. Tokyo, 2000, pp.333-353 (in Japanese)

12. Perry J, Judith M Burnfield : Gait analysis. Normal and pathological function. $2^{\text {nd }}$ ed, chapter 24, SLACK Incorporated, Thorofare, 2010, pp.483-518

13. UcEY, Follett KA : Deep brain stimulation in movement disorders. Semin Neurol 22(2) : 170-182, 2007

14. Krauss JK : Surgical treatment of dystonia. Eur J Neurol 17(1) : 97-101, 2010

15. Botzel K, Kraft E : Strategies for treatment of gait and posture associated deficits in movement disorders : the impact of deep brain stimulation. Restor Neurol Neurosci 28(1) : 115-122, 2010

16. Johnsen EL, Mogensen PH, Sunde NA, Ostegaar K : Improved asymmetry of gait in Parkinson's disease with DBS : Gait and postural instability in Parkinson's disease treated with bilateral deep brain stimulation in the subthalamic nucleus. Mov Disord 22(4) : 590-597, 2009

17. Page D, Butler A, Jahanshahi M : Quality of life in focal, segmental, and generalized dystonia. Mov Disord 22(3) : 341-347, 2007 